\title{
Finite Wave Modeling of Extended-Tube Resonators
}

\author{
Y. Sathyanarayana and M.L. Munjal \\ Facility for Research in Technical Acoustics, Department of Mechanical Engineering, Indian Institute of Science, Bangalore \\ 560012 , India
}

Finite wave analysis of hot exhaust systems involves two modified Riemann variables and an entropy variable. These three variables move along their characteristic paths in the three characteristics approach of the method of characteristics. This paper addresses the modeling and simulation of extended-tube resonators in the time domain with the three characteristics approach. There are two known variables and seven unknown variables across an extended-tube resonator junction. Mass continuity, energy, momentum and entropy equations along with compatibility equations are simultaneously solved by means of the Newton-Raphson method to evaluate the unknown variables. The extended-tube expansion chamber is analyzed by means of the two characteristics approach as well as the three characteristics approach. The simulated results obtained by the two approaches are compared.

\section{INTRODUCTION}

In finite wave analysis, the governing equations are solved in the time domain. The equations governing the unsteady flow in a duct are hyperbolic. The most popular technique for solving these differential equations numerically, is the method of characteristics. Any characteristic variable can be traced in two ways. One is the method of wave diagrams as enunciated by Rudinger, ${ }^{1}$ which has been called a nonmesh method by Benson. ${ }^{2}$ In this paper, it has been called a moving frame method., ${ }^{3,4}$ The other method is Benson's mesh method, ${ }^{2}$ termed here as the stationary frame method..$^{3,4}$

In the moving frame method, certain values of the characteristic variables entering the duct are chosen, usually after each fixed time interval and then their evolution is tracked in terms of the new positions that they occupy after one time step of computation. It is clear that the position or the spatial coordinate of the characteristic variable keeps undergoing change. In other words, the frame of the characteristic variables moves in space. Hence the name given: moving frame method. By way of contrast, in the stationary frame method, certain fixed spatial locations are chosen in the duct and the evolution of the characteristic variables is recorded by assigning newer values to the particular characteristic variable after each time step. Again, it can be seen that newer values are assigned at fixed positions in space. Hence the name given: stationary frame method.

It is well known that in the case of one dimensional unsteady fluid flow, which is adiabatic and reversible, the characteristic variables remain constant along the characteristic curves. Therefore, these variables are called Riemann invariants. In the case of an isentropic flow, their number is two. One of them is associated with the forward wave and the other one with the reflected wave. Hence, in this case, the approach is termed the two characteristics approach $(M O C-2)$. However, when this method is applied to more general hot flow problems, where entropy variations can be large at area discontinuities, etc., the Riemann invariants no longer retain their invariant character and are therefore called modified Riemann variables. In this more general flow case, the number of characteristic variables is three and the third variable is known as the entropy variable. Therefore, in this case, the approach is termed the three characteristics approach $(M O C-3)$. These variables, in their nondimensionalized form, are denoted by $P, Q$ and $A_{0}$ in this paper. Significantly, the two characteristics approach, in general, is not a special case of the three characteristics approach. The method of characteristics is given in Appendix A in brief.

The frequency domain modeling is simple and ideally suited for rapid analysis of a muffler configuration. However, it requires a prior knowledge of the source characteristics, the evaluation of which remains a challenge. In comparison, finite wave modeling by means of the method of characteristics is complete in itself, but is much more cumbersome. The two characteristics stationary frame method has been developed for some complex muffler elements like extended-tube resonators and perforated elements, whereas the three characteristics solution has been limited to simple uniform pipes and simple area discontinuities in the stationary frame method. The model has been primarily developed and used for thermodynamic performance evaluation and not for the aeroacoustic performance evaluation of the exhaust system.

The finite wave analysis of mufflers in the existing literature has been limited so far to a few simple muffler elements. Literature is available only for uniform pipes and simple area discontinuities in both the two and three characteristics approach. $^{2-6}$ The analysis of complex mufflers such as the extended-tube resonator and perforated elements with full nonlinear analysis, i.e., the three characteristics approach is not available in the literature. To be precise, the analysis of extended-tube resonators, such as a three-way junction or simple branch system, was carried out first by Benson et al. in $1963 .{ }^{7}$ However, the computational method used was quite involved because the entropy variable was considered in a moving frame. In this paper one of these lacunae is sought to be filled up inasmuch as extended-tube resonators are simulated in the finite wave analysis making use of the fixedframe method for all variables including the entropy variable. 